\title{
Huanca Urco: Un sitio de control administrativo local, distrito Huancas, provincia Chachapoyas, región Amazonas.
}

\section{Huanca Urco: A local administrative control site, Huancas district, Chachapoyas province, Amazonas region.}

\author{
Luisa Karina Reyes Rodríguez
}

\section{RESUMEN}

EL objetivo de esta investigación se determinó la prospección y reconocimiento del sitio, su estructura del estudio se definió en la metodología arqueológica aplicada en el método de prospección técnicamente en la observación y registro, posteriormente se obtuvo como una interpretación del sitio arqueológico Huanca Urco. El estudio del sitio Huanca Urco tuvo con finalidad de registrar los elementos arquitectónicos y asociaciones que me permitió comprender la ocupación del área de estudio, que se definió con dos aterrazamientos conjuntamente con una estructura rectangular cada uno, dicho tipo arquitectónico es en forma espiral y un rasgo distintivo con respecto a su función de control, llevando a cabo una interrelación de redes de comunicación local. Al mismo tiempo existe la influencia del periodo formativo que contiene por sus características arquitectónicas; y posteriormente inca con la cercanía al camino prehispánico que se ubica en el Noreste del sitio, además se concluye que posiblemente tiene la actividad de control administrativo local por la estratégica de su ubicación.

Palabras clave: Prospección, aterrazamientos, control administrativo.

\begin{abstract}
The objective of this investigation was to determine the survey and recognition of the site, its structure of the study was defined in the archaeological methodology applied in the method of prospecting technically in the observation and registration, later obtained as an interpretation of the archaeological Huanca Urco. The study of the site Huanca Urco had the purpose of registering the architectural elements and associations that allowed me to understand the occupation of the study area, which was defined with two terraces together with a rectangular structure each, said architectural type is in a spiral form and a Distinguishing feature with respect to its control function, carrying out an interrelation of local communication networks. At the same time there is the influence of the formative period that contains by its architectural characteristics; And later inca with the proximity to the preHispanic road that is located in the Northeast of the site, in addition it is concluded that possibly has the activity of local administrative control by the strategic of its location
\end{abstract}

Keywords: Prospecting, terraces, administrative control. 


\section{INTRODUCCIÓN}

El departamento de Amazonas presenta una diversidad de microclima manifestando la complejidad cultural y etnográficas, por lo que ha sido escenario de muchos investigaciones de distintas naturaleza que con lleva a conocer nuestra historia como los grupos étnicos como los Wampis y Awanjún que siguen perdurando en el tiempo, a pesar que la Amazonia ha sufrido modificaciones en forma geográfica-ambiental, social-cultural.

Es importante resaltar que nuestra región es rica en recursos culturales arqueológicos por lo que es necesario conservar y difundir, además de la investigación científica que nos permite entender y explicar su desarrollo en la sociedad.

En los escenarios de las actuales huancas, es una población importante desde épocas prehispánicas en lo cual se detalla en forma etnohistoria:

Según el investigador Narváéz Vargas (2015) los Huancas de Amazonas nos refiere que en la época en la que surgen personajes tan importantes en la historia cusqueña con Mama Huaca, distinguiéndose ésta como una mujer de gran valor que hizo frente al invasor chanca demostrando coraje y actitud despoblada, Estas circunstancias permitieron además el surgimiento de Pachacutec, el gran arquitecto de la ciudad sagrada del Cusco, que devino en ombligo del mundo al momento en que se logra no solo el triunfo guerrero frente a los chancas, sino el inicio de un proceso de expansión que forjó los límites del imperio incaico,tan al norte como el actual territorio de Ecuador, como por el sur,el territorio andino del noreste argentino y el territorio chileno hasta la frontera del rio Maule. De acuerdo con Guaman Poma, fueron los capitanes Ynga Maytac e Inga Urcon los que tuvieron a su cargo las campañas militares de Chancas y huancas "grandes valeroso y grandes capitanes esforzados .fue hijos de Yauar Uacac Ynga ...(Guaman Poma ,1615,156). Por lo tanto el investigador nos da conocer la influencia inca la expansión del territorio norte de ecuador, Noroeste de argentina y chile

Los vecinos más importantes de los chancas, fueron sin duda los grupos organizados bajo la identidad huancas, que tuvo siempre su espacio central en el valle Mantaro, como se le conoce hoy. Uno de los lugares más importantes del territorio andino, no solo por su amplitud, sino por la presencia de agua permanente, permitiendo el desarrollo de una de las más importantes civilizaciones de la sierra central, con poblaciones que cruzaron los Andes y llegaron hacia este espacio privilegiado por la naturaleza (Mattos Mendieta, 1978). La importancia que nos da las organizaciones de los Chancas que dejaron parientes huancas de la zona Amazonas por la expansión que tuvieron concurrieron.

Los huancas tienen una historia muy larga ,que se inicia en los periodos de cazadores y recolectores, alrededor de 7000 años de antigüedad, en sitios como Tuntaya, un abrigo rocoso que arrojo una antigüedad de 4850 años a.C.( (Mallma Cortéz, 1996) teniendo en cuenta que estos sitios han tenido una cronología pre cerámico en relación con los sitios de Cuspinique.

Mediante las investigaciones arqueológicas de la provincia de Chachapoyas; sea efectuado una investigación bibliográfica realizada en la región Amazonas, en general, se ha concentrado mayormente en la cuenca del Utcubamba (abarcando tanto la provincia de Chachapoyas como la de Luya), donde a partir de la existencia de arquitectura monumental como la de Kuelap, así como de las estructuras funerarias o chullpas, son las áreas que más han acaparado la atención de los investigadores, esto se demuestra, a su vez, a nivel regional en la cantidad de sitios (526), reportados a la fecha por el Proyecto Qhapaq Ñan, Temporada 2005, donde sólo en dos provincias (Chachapoyas y Luya) suman 415 sitios, a diferencia de las otras cinco provincias (Bongará, Utcubamba, Rodríguez de Mendoza, Bagua y Condorcanqui) que suman sólo 111 sitios citados en la bibliografía. (Montoya V., Mary.; Zavaleta U.,Gabriela., 2005)

En relación al reporte del Proyecto Qhapaq Ñan 2007, donde se realizan el reconocimiento y prospección en la Cuenca Utcubamba, en las sub cuencas del Sonche margen izquierda (San Francisco de Daguas), Olía margen izquierda y derecha y Ventilla en su margen derecha (Molinopampa). (La Torre \& Campos, 2007)

El sitio arqueológico Huanca Urco presenta en forma espiral con una funcionalidad de control administrativo.

\section{MATERIAL Y MÉTODOS}

El área de estudio del sitio arqueológico Huanca Urco, ubicado en una parte de una loma del mismo nombre, coordenadas UTM siguientes: Norte: 9321694; Este 0181703, y un altura de 2742 m.s.n.m., aproximadamente 10 kilómetros en el distrito de Huancas, provincia de Chachapoyas, región Amazonas.

El trabajo se realizó en dos etapas: la prospección del área y el trabajo de gabinete realizado en octubre a diciembre del 2016. 


\section{Prospección}

Consistió en las dos técnicas, la observación del sitio (exploración) y recorrido del área arqueológica (descripción) con la finalidad de describirlo con exactitud, y el recorrido del sitio.

Es de gran magnitud dándonos cuenta que la par0te principal es la forma de circunferencia sinuosa que se encuentra en la cima o loma del cerro es decir un aterrazamiento que se halla en primer nivel y al mismo tiempo se encuentra el segundo nivel, consistió en cortar parcialmente y en forma técnica las coberturas vegetales en: bejucos, huicundos, zarzamoras entre otras plantas pequeñas, que se encontraban cubriendo en gran manera a las evidencias arquitectónicas. El trabajo de limpieza se hizo usando machete y maderos largos "horcones" este último para arrastrar la vegetación trozada a un solo lugar.

\section{Estudio de Planimetría del área de Huanca Urco (triangulación):}

La triangulación es un procedimiento que se usa para hacer el levantamiento topográfico de una estructura en un mismo nivel por ello solo se tomó las medidas de los muros perimetrales exteriores más no de la estructura central o la que se halló más elevada. El proceso que se llevó a cabo tomando un eje que se encontraba al norte en donde se determinó dos puntos (A y B) con una distancia fija y nivelada. Se ha triangulado un punto que se encontraba al frente del recinto $n^{\circ} 1$ que es el recinto exterior que se nota al ingresar el sitio. Este era parte de un muro que estaba cubierto por rocas, luego de haber tomado la forma de las piedras encontradas en la superficie se procedió a colocar puntos base a una equidistancia de 5 metros para así poder constituir triángulos isósceles que nos permitan poder configurar el muro perimetral del aterrazamiento del segundo nivel, en el lado oeste del sitio se estableció este punto de equidistancia en un metro. Con estos procedimientos se pudo obtener las características arquitectónicas del sitio.

El sitio arqueológico Huanca Urco tiene una aproximadamente una $30 \mathrm{~m}$ de diámetro y el edificio concéntrico tiene $12 \mathrm{~m}$, por lo total tiene un área de 1 hectárea aproximada.

\section{Gabinete}

Se realizó en el Instituto de Investigación de Arqueología y Antropología "Kuelap" de la Universidad Nacional Toribio Rodríguez de Mendoza de Amazonas, donde se registró y catalogó las fotografías en diferentes panoramas y detalles del sitio.

Y procesamiento de datos obtenidos en campo.

\section{RESULTADOS}

El sitio arqueológico de Huanca Urco es un lugar que se encuentra en la cima de un promontorio rocoso con un muro de contención en todo el cerro Huanca Urco, en el primer aterrazamiento se muestra en un primer momento con estructuras que contenga patios circulares a su alrededor, pero después de la prospección eran simplemente patios circulares de construcción moderna por su tipo de mampostería solo puesta sin ningún tipo de argamasa es por eso que solo se tomó en cuenta la parte de la estructura circular sinuosa que tenía un recinto cuadrangular en el lado Noreste del sitio que se encuentra en el primer nivel. Y el segundo aterrazamiento se encuentra en el segundo nivel donde se halla un recinto rectangular con un vano de acceso hacia el Sur, y una hornacina al Noroeste.

La forma del edificio de Huanca Urco en general en forma espiral que aparentemente se accedía por el Norte, el acceso colapsado permitía una vía de tránsito en forma de $\mathrm{L}$ que conectaba con el lado Noroeste del edificio, en este lado del edificio posiblemente hubo un corredor que accedía a la parte superior del edificio que debió ser la parte más importante y restringida del sitio lo que sugiere algún tipo de estatus de los pobladores.

Al mismo tiempo se considera que el material de construcción la piedra se ha extraído de una cantera próxima al sitio que se encuentra en la falda del cerro para la construcción y al camino prehispánico; donde el camino luce secciones de piedra caliza incluyendo tramos con escalones dado la fuerte pendiente del terreno.

\section{Estructuras Arquitectónicas}

El muro perimétrico o contención es forma circular sinuosa con una altura de $3.20 \mathrm{~m}$. de altura con piedras labradas y no labradas ; en la parte Noreste que se encuentra la estructura cuadrangular, en mal estado de conservación; y en el nivel alto de loma del cerro se halla resto arquitectónico de una sola estructura de planta rectangular 


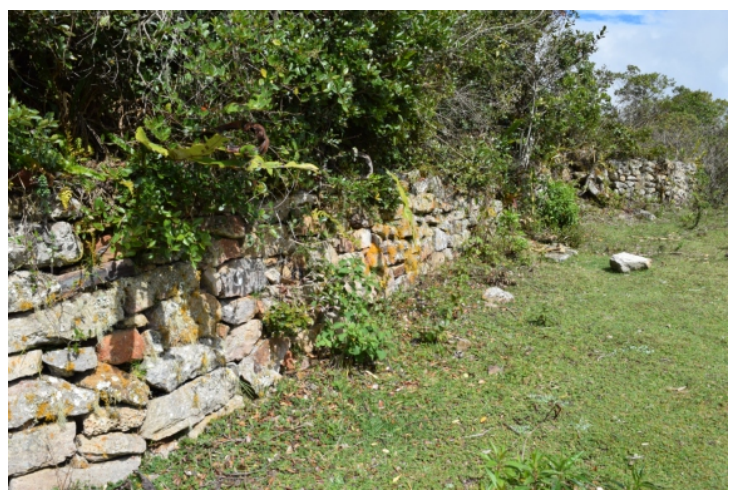

Figura 01. Muro de contención sinuosa en el nivel bajo del sitio Huanca Urco.

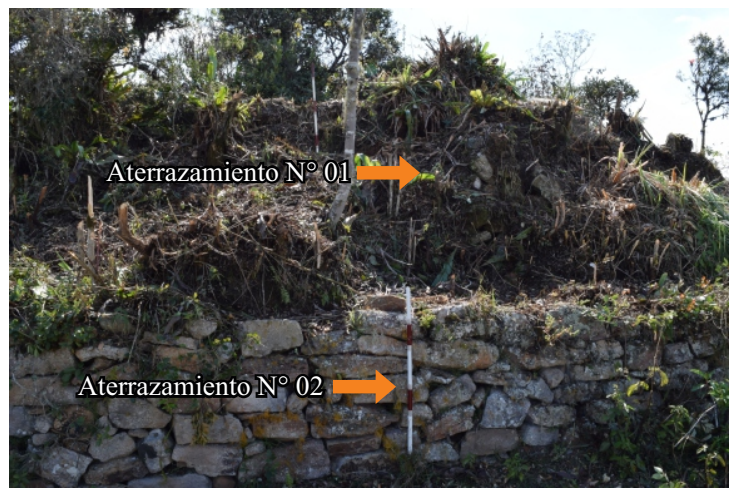

Figura 02 . Aterrazamiento $\mathrm{N}^{\circ} 01 \mathrm{y} \mathrm{N}^{\circ} 02$ del sitio Huanca Urco.

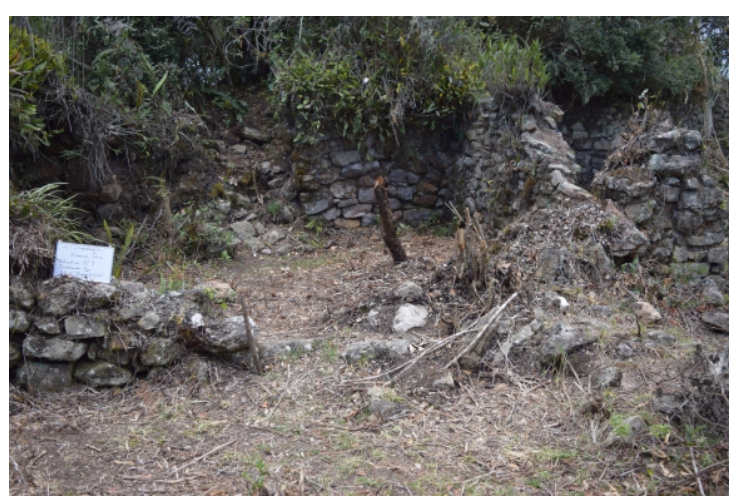

Figura 03. Estructura cuadrangular en el nivel bajo del sitio Huanca Urco.

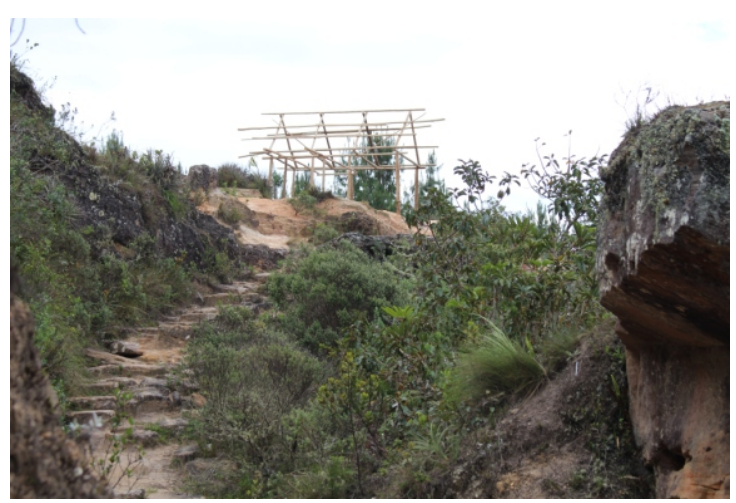

Figura 04. Camino pre hispánico en la parte intermedia del cerro Huanca Urco.

\section{Materiales y Técnicas Constructivos \\ Materiales de Construcción}

El sitio arqueológico de Huanca Urco, presenta diferentes elementos constructivos para sus edificaciones arquitectónicas de sus estructuras estos son: piedras, pachillas y argamasa. Estos materiales se identifican en el nivel superficial. Además presenta relleno de piedras y tierra, que fueron nivelados, al mismo se observa pachillas que cumplen la función de cuñas dentro del mortero de barro en las juntas de las piedras labradas para una nivelación horizontal de una estructura.

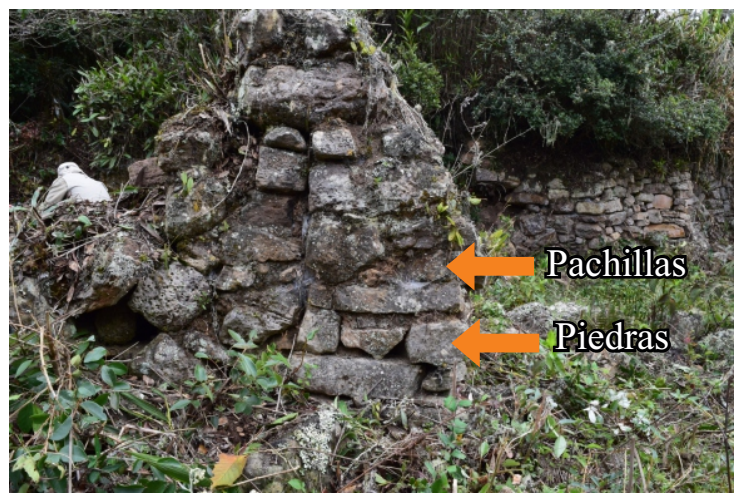

Figura 05. Detalle de materiales de construcción de la estructura del sitio Huanca Urco.

\section{Elementos Constructivos y Sistema Estructural}

Es una parte física que se integra a una obra de construcción como tenemos en el sitio de Huanca Urco.

\section{Escalinata:}

El sitio Huanca Urco está conformando por escalinata de diferentes dimensiones $0.90 \mathrm{~m}$. de ancho por una altura de $2.50 \mathrm{~m}$. compuesta por piedra caliza en regular tamaño formando escalones, que da al acceso del nivel superior o alto que se encuentra la estructura rectangular.

\section{Ventana}

Se halla en la estructura rectangular en el primer aterrazamiento, contiene una (1) ventana de $0.40 \mathrm{~m}$. de lago por $0.40 \mathrm{~m}$. de ancho. Esta un regular estado de conservación. 


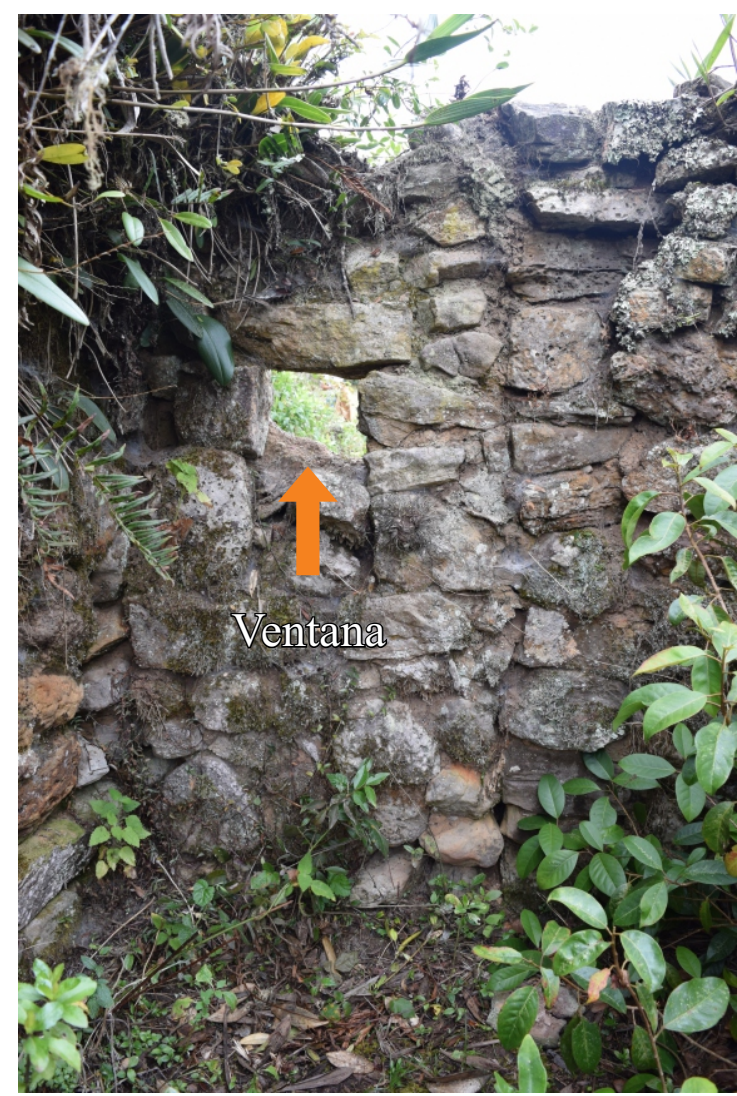

Foto $\mathrm{N}^{\circ} 10$. Detalle de una ventana en la estructura cuadrangular en el aterrazamiento bajo del sitio Huanca Urco

\section{DISCUSIÓN}

El sitio arqueológico Huanca Urco está en una cima del cerro Huanca Urco y se encuentra en una zona de selva o ceja de selva son moderadamente profundos y arcillosos saturados de humedad, debido a las frecuentes precipitaciones fluviales. Asimismo por tratarse de una zona de exuberante vegetación y de una gruesa capa de descomposición vegetal ha permitido guardar gran concentración de humedad, lo cual ha hecho colapsar las paredes de los muros y con el crecimiento de vegetación de grandes raíces ha contribuido a destruir evidencias de los materiales culturales. Las estructuras que se encuentran colapsadas, debido también a las prácticas agrícolas o ganadera de años atrás y que ésta ha alterado los contextos arqueológicos.

El sitio arqueológico Huanca Urco es una diferencia en los demás sitios arqueológicos, por tener aterrazamientos en 2 niveles en diferentes dimensiones que atribuyeron a no deslizarse el terreno; la arquitectura del sitio es de forma espiral lo cual contiene en el nivel bajo una forma circular sinuosa y a la vez adosada con una estructura cuadrangular y en el nivel alta se encuentra una estructura rectangular, teniendo la posibilidad que este sitio haya sido un lugar de control administrativo local cerca al camino prehispánico que se encuentra en la parte Noroeste del sitio arqueológico Huanca Urco. Y a la vez resaltar la relación que tiene el camino prehispánico que esta direccionado hacia el Sur con la actual ciudad de Chachapoyas y luego con todos los pueblos de alto Utcubamba interrelacionándose en los diferentes contactos y lugares de los sitios arqueológicos que cuenta y que no son investigados aún.

El sitio arqueológico Huanca Urco por ser una estructura sencilla y simple se podría considerar que pertenece un periodo temprano, dando lugar que en otros lugares arqueológicos de la región Amazonas es diferente y más compleja arquitectónicamente, por lo que algunos investigadores han propuesto que Huanca Urco tiene filiación Formativo de la zona del Centro del Perú como Ataura en Jaula y San Blas en Ondores quedando la posibilidad que la ocupación del sitio Huanca Urco no se encuentre dentro de la ocupación de la Cultura Chachapoyas y por ello se debe implementar un proyecto de investigación arqueológica para confirmar esta posible evidencia cultural.

\section{CONCLUSIONES}

El sitio arqueológico Huanca Urco como evidencia de su conjunto de estructura respondería al periodo temprano cuya funcionalidad sería a un centro administrativo local, por la estratégica ubicación y al mismo tiempo la relación con el camino prehispánico, teniendo en cuenta la jerarquía que ejercía en esta zona, por ello es necesaria continuar con las futuras investigaciones para contrastar lo sostenido en el texto.

\section{AGRADECIMIENTOS}

La autora agradece a las autoridades de la de la Universidad Nacional Toribio Rodríguez de Mendoza de Amazonas y el Instituto de Investigación de Arqueología y Antropología "Kuelap"; a los estudiantes y docente de la escuela profesional de Arqueología de la UNTRM por el esfuerzo académico y físico durante los trabajos de prospección dando el énfasis de las investigaciones arqueológicas que se desarrolla en nuestra región Amazonas y a la Alcaldesa Distrital de Huancas Sra. Deysi Solano Cotrina por su ayuda desinteresada en los trabajos de Investigación en la Amazonia Peruana. 


\section{REFERENCIAS BIBLIOGRÁFICAS}

La Torre, E., \& Campos, C. (2007). Inventario Nacional de Sitios Arqueologicos Macro Región Norte.Región Amazonas. Instituto Nacional de Cultura.Proyecto Qhapaq Ñan.

Mallma Cortéz, A. (1996). Introduccion a la Arqueología e Historia de los Xauxa-Huancas. Seminario de Historia Rural, Universidad Nacional Mayor de San Marcos. Lima.

Mattos Mendieta, R. (1978). Primeras sociedades sedentarias del Mantaro. III Congreso del Hombre y la Cultura Andina., (págs. 265-293). Lima.

Montoya V., Mary.; Zavaleta U.,Gabriela. (2005). Inventario Nacional de Sitios Arqueologicos Macro Región Norte.Región Amazonas. Lima.: Instituto Nacional de Cultura.

N a r vá e z Vargas, A. y. ( ( 2 $\left.\begin{array}{llll}2 & 0 & 0 & 5\end{array}\right)$. Investigaciones, Conservación y Acondicionamiento Turístico de la Fortaleza de Kuelap.Etapa III. Chachapoyas: Gobierno Regional de Amazonas.

Ruíz Estrada, A. (1969). Alfareria del estilo Huari en Kuelap. En Boletin del Seminario de Arqueología, $\mathrm{N}^{\circ} 4$. Lima. 